\title{
LE DELTA DU DANUBE ET SES RICHESSES ${ }^{(1)}$
}

\author{
(Suite et fin) \\ par George BARCA \\ Ingénieur, Docteur, Agrégé \\ Professeur Polytechnique de Galatzi -- ROUMANIE
}

L'application d'une pisciculture rationnelle dans le Delta proprement dit (Delta fluvial) repose sur le maintien d'un régime d'inondations naturel.

Le production piscicole du Delta s'est avérée capable d'atteindre aisémient $100 \mathrm{~kg} / \mathrm{ha} / \mathrm{an}$ dont $75 \mathrm{~kg} / \mathrm{ha} / \mathrm{an}$ sont constitués par la carpe (Cyprinus carpio).

Afin de peupler les lacs avec des carpes, on a aménagé sur les terrains secs de nombreux élevages, produisant la quantité d'alevins susceptible de suppléer à la diminution de celle obtenue jusqu'à présent par voie naturelle, dans ies zones de reproduction situées le long du fleuve jusqu'aux Portes de Fer; à l'heure actuelle, ces zones ne font plus partie du circuit piscicole, étant rendues à l'agriculture, par les endiguements réalisés. présent

Dans le Delta proprement dit, deux problèmes capitaux se posent à

- l'accroissement de la production piscicole;

- la protection des lacs et de leur existence.

Le premier prós!ème concerne le maintien d'un mélange naturel des espèces et l'amélioration de ce mélange par une pêche sélective, afin d'éliminer les rapaces et particulièrement le brochet (Esox lucius). dans les zones au régime libre d'inondations, ainsi que l'amélioration des indices de production par la sélection du cheptel piscicole existant dans les lacs.

Pour la protection des lacs contre le colmatage naturel, provoqué par les alluvions du Danube, on a entrepris des recherches expérimentales à

(1) Voir BULLETIN FRANÇAIS DE PISCICULTURE, n 238 du 30 septembre 1970. 
pied d'œuvre, afin d'étudier la possibilité d'assainir par colmatage dirigé les terrairı embourbés, faiblement productifs, se trouvant encore à un régime raturel a'inondations. Le colmatage de ces terrains, situés sur la ligne périphèrique des complexes piscicoles du Delta proprement dit, aura pour résultat leur fertilisation et leur intégration dans l'économie agricole, zootechnique et piscicoie.

Par le colmatage dirigé des zones à faible productivité, on obtiendra indirectement la diminution de l'embourbement des lacs par des alluvions et, impliciiement, une prolongation de la vie de ces lacs. Le procédé utilisé est nouveau at l'on espère qu'il menera à une meilleure utilisation dee ressources fertilisantes des eaux du Danube.

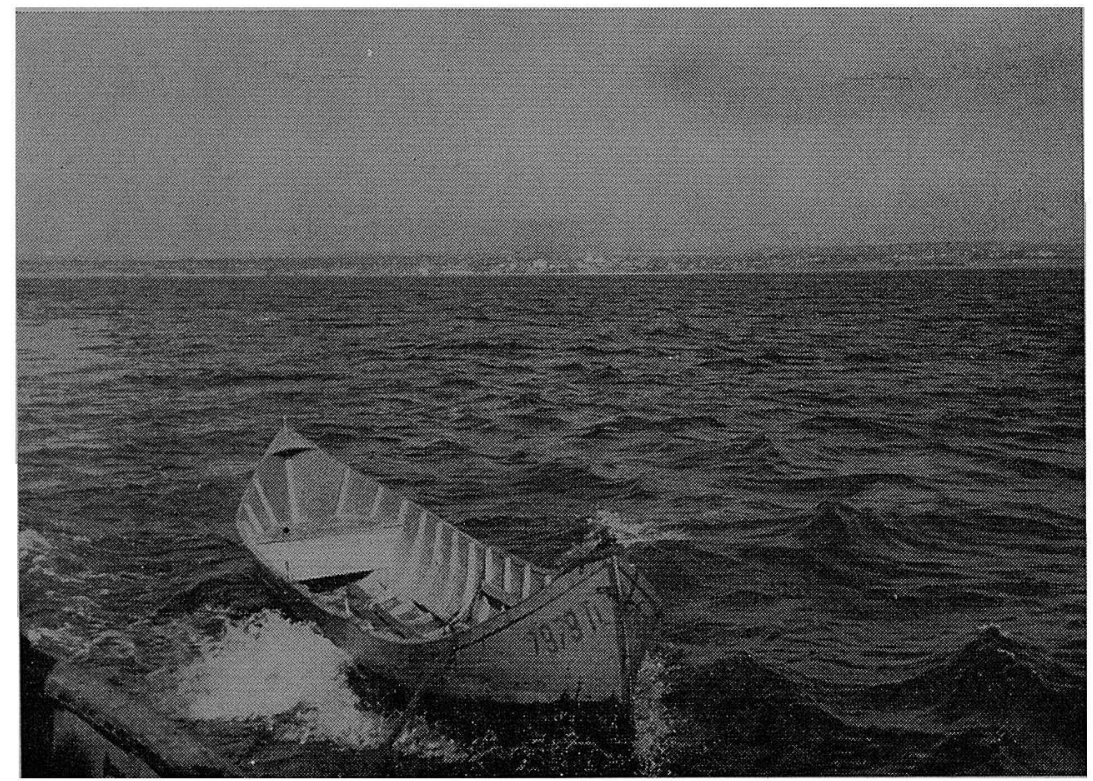

Delta du Danube : le lac Razelm

Comparée à celle du Delta proprement dit, l'exploitation piscicole préconisée dans le système lagunaire Razelm-Sinoe diffère dans une grande mesure, par l'effet du caractère spécifique de ces lagunes.

Ce groupe de lagunes détient un potentiel de production beaucoup plus élevé que sa production piscicole actuelle.

La cause principale de ce fait réside dans sa population piscicole très inférieure, malgré les grandes stations d'alevinage aménagées dans la zone nord du complexe et qui ont commencé à fournir l'alevin de carpe sélectionné, destiné à peupler le lac Razelm.

Les problèmes qui n'ont pas encore èté résolus pour ce système de lagunes sont les suivants:

- trouver une formule idéale pour mettre en valeur son riche potentiel de nourriture, caractéristique des eaux saumâtres ; 
- maintenir un équilibre de la salinité correspondant à la formule de peuplement de chaque compartiment lagunaire séparément;

- établissement des procédés hydrotechniques à appliquer pour consolider le cordon littoral à l'endroit du compartiment Sinoe, afin de prolonger son existence car, sous l'action continue des courants marins, il tend à disparaitre comme il y a 2000 ans, lorsque l'ancienne ville d'Histrie était un port de la Mer Noire, sur les rivages du golfe qui existait à cette époque.

Afin d'augmenter la production piscicole du compartiment Razelm, des expériences sont en cours concernant l'acclimatation d'espèces importées de République Populaire Chinoise, se nourissant de plancton et de mollusques. Pour certaines de ces espèces, on a déjà obtenu des indicateurs sur la prolificité et la maturité des reproducteurs ainsi que sur les processus de fécondation, d'éclosion et de survivance en différentes phases.

En appliquant les mesures sus-mentionnées, on escompte obtenir des productions d'au moins $300 \mathrm{~kg} / \mathrm{ha}$ en rég!me naturel, contre $100 \mathrm{~kg} /$ ha que l'on obtient aujourd'hui.

\section{***}

Quant à la production piscicole du chenal des trois bras du Danube - Chilia, Sulina, St. Gheorghe - elle sera améliorée par une péche sélective, afin de limiter la proportion des rapaces qui ont une influence négative sur la production des complexes du Delta.

En même temps, on protégera les espèces ayant une valeur économique supérieure, par des mesures visant à les épargner et à peupler les lacs avec l'alevin correspondant; ces mesures sont d'autant plus nécessaires que le chenal du Danube sera divisé à l'avenir par les barrages des grandes centrales hydro-électriques danubiennes, semblables à celle des Portes de Fer. Dans ce complexe de mesures, on examinera avec un soin tout particulier le problème des Acipensérides dont le stock décroit dans le bassin de la Mer Noire chaque année, par l'effet de la pêche excessive pratiquée dans le Delta et surtout dans la zone des embouchures du Danube, là où l'alevin reproduit dans le fleuve devient la proie des engins de pêche en automne, lorsqu'il revient à la Mer Noire.

Quoiqu'actuellement, sur le territoire de la Roumanie, y compris les eaux territoriales de la Mer Noire, on pratique les mesures de protection établies dans le cadre de la Convention entre les pays riverains du Danube, bien des efforts seraient encore nécessaires.

$\because \quad$ En dehors de l'action visant à faciliter la migration vers les endroits où le poisson trouve sa nourriture.. et pond ses œufs et la construction de passages dans le corps du barrage même, on procédera à l'organisation de stations d'alevinage, productrices d'esturgeons, ainsi qu'à l'application de mesures sévères pour protéger, d'une part, les reproducteurs dans la zone d'accès de la Mer Noire, aux embouchures du fleuve et, d'autre part, des alevins qui abandonnent chaque année le Danube pour la Mer Noire, en vue de leur maturité.

Parallèlement aux esturgeons, les harengs du Danube détiennent une grande importance. Cette espèce quitte chaque printemps la mer pour pondre dans les eaux du Danube; l'alevin revient en automne à la mer après avoir consommé l'abondante nourriture des eaux du fleuve. 
Le problème du roseau - commun à tous les deltas - doit être résolu en concordance avec l'économie piscicole, extrêmement sensible à la récolte mécanisée. II sera donc utile d'examiner les problèmes suivants :

- La récolte mécanisée du roseau devra utiliser des outillages dont la pression exercée par le véhicule récolteur soit inférieure à la portance biologique du terrain, afin de ne pas écraser les rhizomes et les bourgeons qui produiront au printemps les nouvelles tiges. A défaut de mécanismes adéquats, capables d'assurer cette condition, ia récolte mécanisée du roseau doit être abandonnée coûte que coûte. En persévérant, on risque de détruire pour bon nombre d'années la nouvelle végétation du roseau, jusqu'à une éventuelle réfection des rhizomes détruits durant une seule campagne de récolte mécanisée.

- Eviter le remplacement du régime naturel d'inondation du Delta par un régime dirigé et des endiguements exigés à l'heure actuelle par la récolte mécanisée du roseau, ce fait empêchant le maintien de l'équilibre biologique du Delta; il freine en effet le développement harmonieux de son évolution naturelle et, par là-même, est défavorable à toutes les autres économies du Delta, y compris celle du roseau.

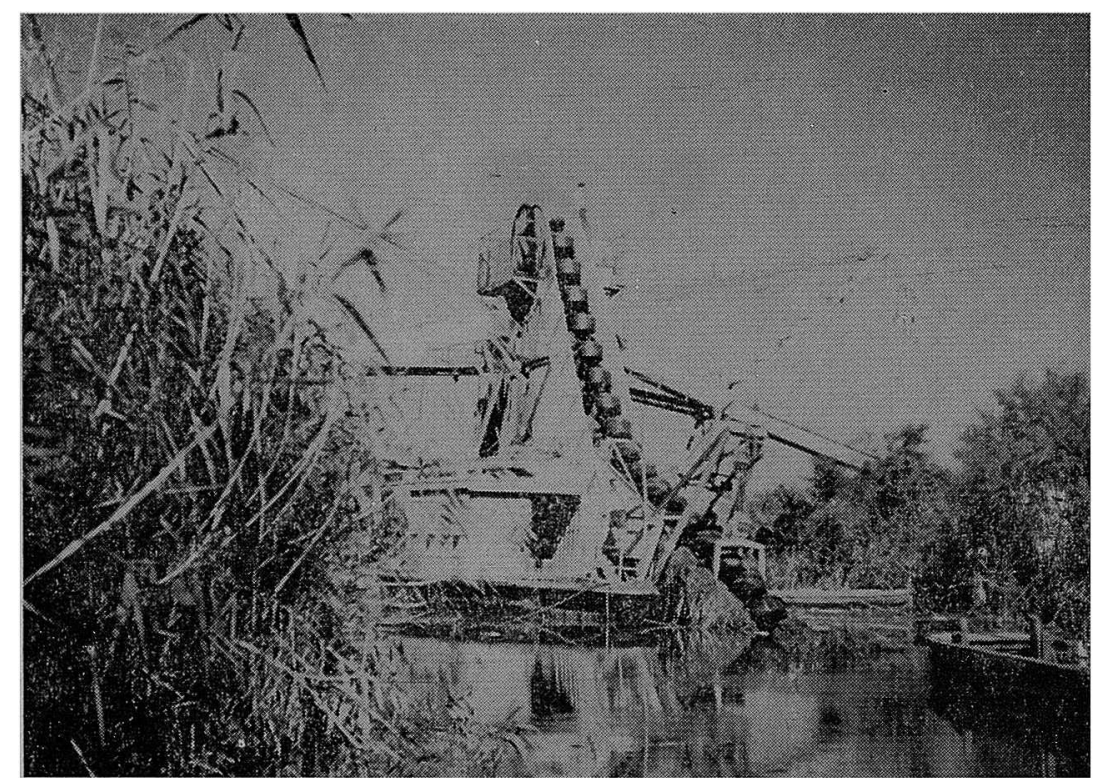

Delta du Danube : construction d'un canal

- Empêcher la construction de routes réclamées par l'exploitation du roseau; en cas de force majeure, les canaux préconisés devront s'intégrer dans les principes d'hydro-améliorations nécessaires à la conservation du Delta. On pourra ainsi éviter une dégradation plus grave des lacs en accélérant le processus de colmatage de ceux-ci et de leur hinterland.

- Eviter autant que possible l'utilisation d'un outillage bruyant.

Afin de rendre possible la solution des problèmes cités, il a été néces- 
saire de créer une direction unique, seule capable de concilier les intérêts multiples de cette région bien particulière.

Compte tenu de ce qui vient d'être dit, il faudra considérer comme principale, l'économie piscicole, toutes les autres étant coordonnées en fonction de celle-ci. De cette manière l'évolution de cet espace du Delta se développera librement, permettant aux nombreux intérêts économiques de s'harmoniser avec les exigences du tourisme - problème actuel et permanent - ainsi qu'avec les recherches scientifiques.

\section{*i*}

L'ensemble de mesures envisageant l'amélioration de la situation économique du Delta ne néglige pas non plus le problème démographique de cette importante contrée de la Roumanie; on envisage une systématisation des villages actuels, car l'habitant du Delta s'est adapté de longue date aux conditions de vie totalement différentes de celles du reste du pays, fes conditions correspondant au genre de travail qu'il accomṕlit toute l'année durant.

D'autre part, le plan d'aménagement du Delta pour la mise en valeur de son potentiel économique, envisage de conserver son pittoresque unique qui rend cette région si différente de l'aspect général du pays.

Parallèlement aux travaux hydrotechniques et d'organisation des différents secteurs d'activité dans le Delta, l'Office National de Tourisme (ONT) intervient, lui aussi, avec des travaux importants afin d'assurer l'accès aux recoins secrets du Delta, véritables jungles où l'homme n'a pas encore pénétré.

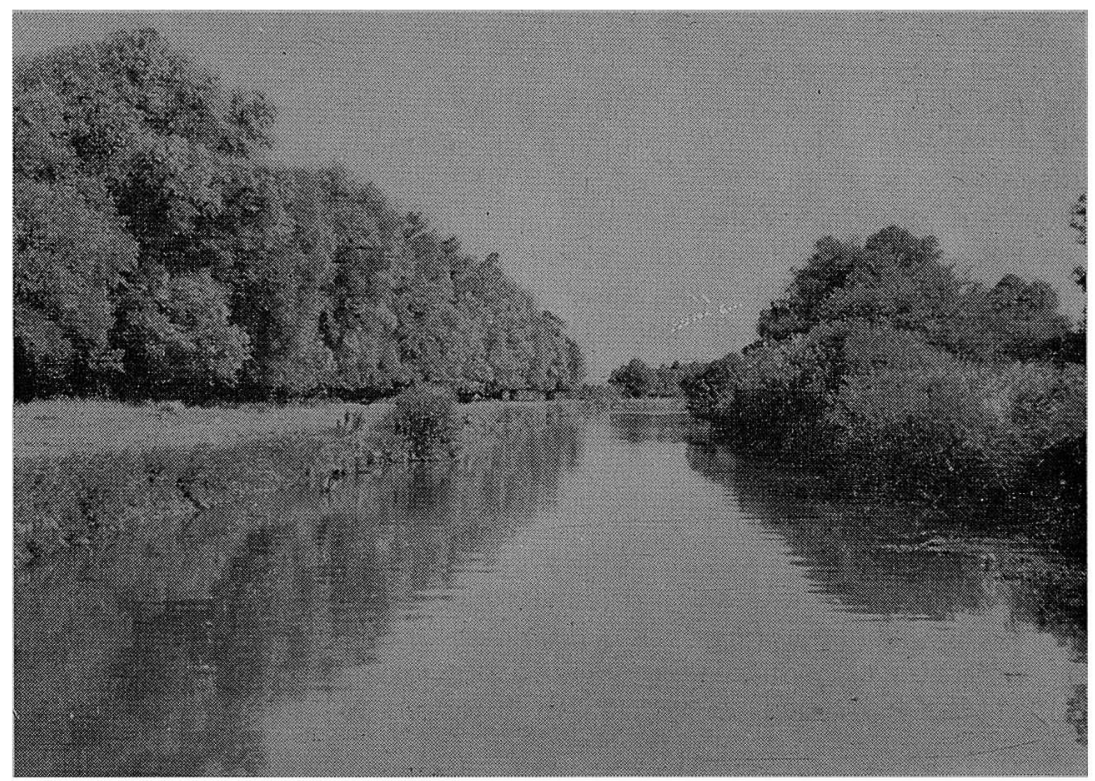

Delta du Danube : un canal navigable

De grands canaux, permettant l'accès des touristes dans des embarcations à moteur, sont entretenus dans les zones caractéristiques du Delta. Des 
pavillons de chasse, des cabanons pour ceux qui viennent y chercher la solitude se mirent dans les lacs ou surgissent au cœur de ces forêts de lianes poussant sur des iles créées, au jour le jour, par les eaux boueuses du Danube, ou bien sur les dunes dont la mystérieuse architecture est l'œuvre millènaire du vent et de la mer.

Dès le port de Tulcea qui monte la garde aux portes du Delta, des hôtels touristiques flottants invitent à un voyage plein de surprises et de paysa. ges enchanteurs dans le royaume des oiseaux migrateurs; c'est le pays du pélican (Peleḱkanus onnocrotalus), de l'égrette (Egreta garzeta), de l'ibis au plumage noir, lointain parent de l'oiseau sacré de l'Egypte (Bubulcus ibis), des cygnes blarics (Cygnus cignus), des canards sauvages au plumage vivement coloré qui reviennent du nord et des grues (Melagosus grus) qui font escale dans le Delta et sur les hauts rivages de la Dobroudja. Volant en automne vers les pays chauds, ils s'arrêtent dans le Delta car, malgré de nombreux changements, il reste le refuge - le dernier en Europe - de tant d'oiseaux migrateurs, certains en voie de disparition.

Pour la protection de ces oiseaux rares dont certains d'un grand intérêt scientifique, on a prévu dans la zone maritime de nombreuses réserves naturelles, couvrant des dizaines de milliers d'hectares. Une vie paisible, à l'abri de toute présence humaine est assurée là à ces éternels voyageurs ailés - parure du Delta et du vieux Danube.

Le Delta du Danube, tout comme les autres deltas du monde, par ses caractéristiques propres, représente une unité hydrographique réclamant un régime d'exploitation tout à fait particulier, différent du reste des eaux.

Du fait que les deltas sont des terrains en cours de formation, dönc en équilibre instable, leur évolution naturelle est extrêmement sensible aux interventions étrangères à leur spécificité. Le déroulement de leur processus naturel de murissement peut être gêné par des actions d'opportunité, dans une mesure tellement grande qu'une bonne partie des avantages qui en découlent disparaissent, sans possibilité de retour et cela par l'effet de l'irréversibilité des processus qui ont lieu dans l'évolution du murissement des deltas. Voilà pourquoi une attention et de soins tout farticuliers doivent être accordés $\dot{a}_{\text {in }}$ l'exploitation de ces "oasis aquatiques" à caractère permanent, capables d'offrir au pays qui les possèdent des bénéfices économiques complexes et supérieurs par rapport à d'autres zones.

En dehors du côté économique de ces régions, le tourisme prèsente une importance sans rival.

Afin d'obtenir une meilleure conservation de ces zones géographiques en permanente métamorphose, il serait peut-être nécessaire d'organiser un Congrès International, ayant la mission de rédiger un plan de mesures d'orientation pour tous les types de deltas, en fonction de leur caractère spécifique.

De cet échange d'idées sur le plan international, il résulterait une ligne de conduite dont l'absence est ressentie chaque jour davantage aujourd'hui si l'on considere l'apport multilatéral des deltas du monde à la vie des peuples au cours de l'histoire. 